(2) Open Access Full Text Article

ORIGINALRESEARCH

\title{
Impact of Social Media and Photo-Editing Practice on Seeking Cosmetic Dermatology Care
}

\section{Harshit Agrawal \\ Sudha Agrawal $\mathbb{D}$}

Department of Dermatology, B.P Koirala Institute of Health Sciences, Dharan, State-I, Nepal
Correspondence: Harshit Agrawal Department of Dermatology, B.P Koirala Institute of Health Sciences, Dharan, State I, Nepal

Tel +977 9842025953

Email harshitagrawaldharan@gmail.com
Background: The use of social media and photo-editing practice has grown enormously over the past decades. Photo editing can alter a person's desire to look better in photographs posted on social media platforms.

Objective: To assess the cosmetic dermatology seeking behavior of social media users and those who edit photographs before posting them on social media platforms.

Methods: A validated self-administered structured questionnaire via Google form was sent to 550 social media users in Nepal. It included 5 sub-headings: use of social networking sites, photo-editing practices, awareness and motivation about cosmetic dermatology care, cosmetic dermatology care seeking behavior and self-esteem.

Results: Facebook and Instagram were the preferred social networking sites for posting photographs. One-fourth of the participants edited $>40 \%$ of the total photos posted in social media. Hiding skin lesions was the most common reason $(36.3 \%)$ for photograph editing. Fifty percent of the respondents felt the need to look better; repair skin damage; be able to look good without make up; look younger; feel happier and improve total quality of life as a "lot and top" motivation for using the cosmetic dermatological procedures. A majority preferred to seek cosmetic dermatology care from non-dermatologists because they felt dermatologist visit was not needed, the services were costly and they could not visit due to their busy schedule. On multivariate analysis, respondents who were aware of skin care favored seeking cosmetic dermatology care from dermatologists.

Conclusion: Higher investment in social media and photo-editing practices might be associated with increased non-dermatologist seeking behavior.

Keywords: cosmetic dermatology care, motivation, photo-editing practice, self-esteem, social media users

\section{Introduction}

Social media use has grown immensely over the past decades with a further spike in the last 12 months. More than half of the world population uses social media, of which, $99 \%$ access them via mobile at some point. About $83 \%$ of the younger age group look for medical information on the internet. ${ }^{1,2}$ The photo editing applications can alter an individual's appearance in photographs posted on social media. Choosing to alter one's appearance means recognizing a personal imperfection, and this repeated behavior may drive an individual to seek dermatologic care for various skin problems. ${ }^{3}$ Some studies have shown that the wide spread use of photo editing can negatively affect body satisfaction and self-esteem. ${ }^{4-7}$ However, a few recent studies showed an increase in dermatological care seeking behavior among social media users who edit their photographs. ${ }^{3,5,8}$ 
However, there are a few challenges in countries like Nepal where the disproportionate dermatologist/patient ratio leads to inadequate dermatological services to the general population. ${ }^{9,10}$ Old dermatologists feel that cosmetic dermatology is not their specialty and there is a rampant influx of medical and non-medical professionals into the dermatology workforce because they feel, performing skin modifications is simple to practice with good monetary gain. ${ }^{11}$ Moreover, people may be influenced by non-dermatological professionals including beauty vloggers for cosmetic dermatology care.

There are no such studies done in Nepal, which can provide the analysis of cosmetic dermatology care seeking behavior of social media users and those who edit their photos prior to posting them on social media. This study assessed the awareness, motivational factors about cosmetic dermatological procedures and cosmetic dermatology seeking behavior of Nepalese social media users to the dermatologists or non-dermatologists.

\section{Materials and Methods}

A self-administered structured questionnaire in Google Forms was sent through social networking sites to undergraduate students of different medical colleges of Nepal from 1st September to 28th September 2020. They were also requested to invite other friends and family members between 18 and 34 years to participate in this survey. Pretesting of data collection tool was done in 40 participants to ensure accuracy and validity of the contents of the information prior to the study participant enrollment. The sample size was calculated on the basis of the previous study $^{3}$ where the expected proportion of social media users seeking cosmetic dermatology care was $41.2 \%$ with a relative precision of $10 \%$ and confidence interval of $95 \%$, a total of 550 participants were enrolled. The study was approved by the Dermatology Departmental Research Unit (DRU) with DRU number: 05/2020 for further implementation and then the ethical clearance was taken from Institutional Review Committee (IRC), BPKIHS. The study implies with the Declaration of Helsinki.

The survey's first page included a description of the study, sociodemographic characteristics of the participant and exclusion criteria. The notification to respondents who continued with the survey was served as informed consent for study participation.

The contextual matter was studied under 5 different sub headings.

\section{Use of Social Networking Site}

The participants were asked to select the social networking site they use (identifies the popular social networking sites in Nepal ${ }^{12}$ ); the number of hours spent per day on these sites; whether the use of social media helped them to be more aware of cosmetic dermatology care or not; had they ever posted a picture of themselves on these sites; the preferred social networking site for posting photographs and number of photos posted per week.

\section{Photo-Editing Practice}

The participants were asked if they ever had edited a photo before posting on social media; the percentage of edited photos posted on social media platforms; average time spent on editing; the reasons for editing; those who selected hiding the skin lesions as the reason for photo editing were asked about the reason and type of skin lesions edited and whether using social media and photo editing made them more conscious about their skin lesions or not. In addition, they were asked whether they ever had untagged or removed a photograph of themselves from social media if the photograph "Was not digitally enhanced or edited to their liking."

\section{Awareness and Motivation About Cosmetic Dermatological Care}

The participants were asked "what according to them was cosmetic dermatology care" and their awareness about the common cosmetic dermatological procedures.

They were also asked about the motivations they thought were important for those cosmetic dermatology procedures like to look better, or more attractive to oneself; repair damage of skin; be able to look good without make up; look younger or fresher; feel happier or better overall; improve total quality of life in a scale of 1-5 (where 1- least, 2- little bit, 3- moderate, 4- a lot and 5top motivation).

\section{Cosmetic Dermatology Care Seeking Behavior of Social Media Users}

The participants were asked about their visit to a dermatologist for their cosmetic dermatology care. They were also asked whether they would ever seek an opinion from a dermatologist for their cosmetic dermatology care or take advice from friends, family or beauty influencers etc. They were also asked about the reasons for not taking advice from a dermatologist (not needed, beauty 
products can hide my problem, fear of procedure, costly, unavailability of the services, busy schedule or ineffective service).

\section{Self-Esteem of the Participants}

Previously validated Rosenberg Self-esteem Scale was used to measure the respondents' self-esteem. ${ }^{13}$ This contained a total of 10 questions and was scored as follows: For question number 1, 2, 4, 6, and 7: Strongly agree $=3$; Agree $=2$; Disagree $=1$; Strongly disagree $=0$ whereas for questions $3,5,8,9$, and 10 (which were reversed in valence): Strongly agree $=0 ;$ Agree $=1 ;$ Disagree $=2$; Strongly disagree $=3$. The score ranged from $0-30$; higher scores indicated higher self-esteem. The score of $0-15$ was considered as low self-esteem and $>15$ as high self-esteem.

The participants were shown appreciation and gratitude for their effort and were also given the contact details of the Department of dermatology for future consultations. Ten participants were randomly rewarded with mobile phone recharge gift cards to encourage them.

\section{Parameter/Variables Measured Independent Variables}

Age, sex, education, occupation, use of social networking sites, use of photo editing applications, awareness of cosmetic dermatology care, motivational factors and selfesteem.

\section{Dependent Variables}

Cosmetic dermatology care seeking behavior.

\section{Data Management and Statistical Analysis}

The data was entered in Microsoft Excel and converted into Statistical Package for Social Science Inc. Chicago, IL., USA version 10.5 for statistical analysis. For descriptive analysis, percentage, ratio, mean, standard deviation, median were calculated along with graphical and tabular presentations. For inferential statistics, bivariate analysis was done using $\chi^{2}$ test, odds ratio and a $95 \%$ confidence interval. Univariate and multivariate logistic regression was done to determine the association of the cosmetic dermatology care seeking behavior of social media users with various independent variables. Odds ratios (OR) significance was determined by the Wald Chi-squared test and predictors with $\mathrm{p}<0.20$ for subsequent multivariate analysis. A p value $<0.05$ was considered statistically significant.

\section{Results}

\section{Sociodemographic Characteristics}

The maximum participants were from 18 to 24 years (460, $83.6 \%$ ) with a mean age of $22.43 \pm 2.665$ years and a female-to-male ratio of 1.24:1. Most of the participants were students $(464,84.4 \%)$ and nearly $66 \%$ of them had completed graduation at least.

\section{Use of Social Networking Sites and Photo-Editing Practice}

Facebook was the most used social networking site $(86.4 \%)$ followed by YouTube (82\%) and Instagram (81.1\%). Approximately half of them used social networking sites for more than 3 hours a day and almost all the participants (97\%) had posted their photos on social media sites. The preferred site for posting their photos was Instagram (53.3\%) followed by Facebook (33.7\%). Around $99 \%$ of the participants posted $<5$ photos per week and $81 \%$ of them had edited a photo before posting. The average time spent by a majority of the participants to edit one photo was less than 5 minutes. About $25 \%$ of the participants edited more than $40 \%$ of the total photos posted on social media. Our study observed that hiding the skin lesion was the most common reason $(36.3 \%)$ for editing a photograph (Figure 1). Of the skin lesions edited, acne or acne scar, pigmentation and dark circle were the most common. Moreover, we also noted that the majority of the participants (64\%) edited the photo because they felt that their skin looked perfect after editing (Figure 2). Maximum participants admitted that using social media (71\%) and photo-editing applications (66\%) made them more conscious of their skin lesions and imperfections. Fifty percent of participants were dependent on appearance and had untagged themselves if the photo posted by others was not edited to their liking.

\section{Awareness and Motivation About Cosmetic Dermatology Care}

Interestingly most of the respondent, (79\%) thought that cosmetic dermatology care deals with both improving the appearance of skin and treating skin disease. Fifty-four percent of participants felt that social media made them more aware of skin care. In our study, $44 \%$ of participants were aware of common cosmetic dermatological procedures, and of them, the maximum knew about acne scarring treatment, hair transplantation and laser for hair removal. More than $50 \%$ of participants felt all the six 


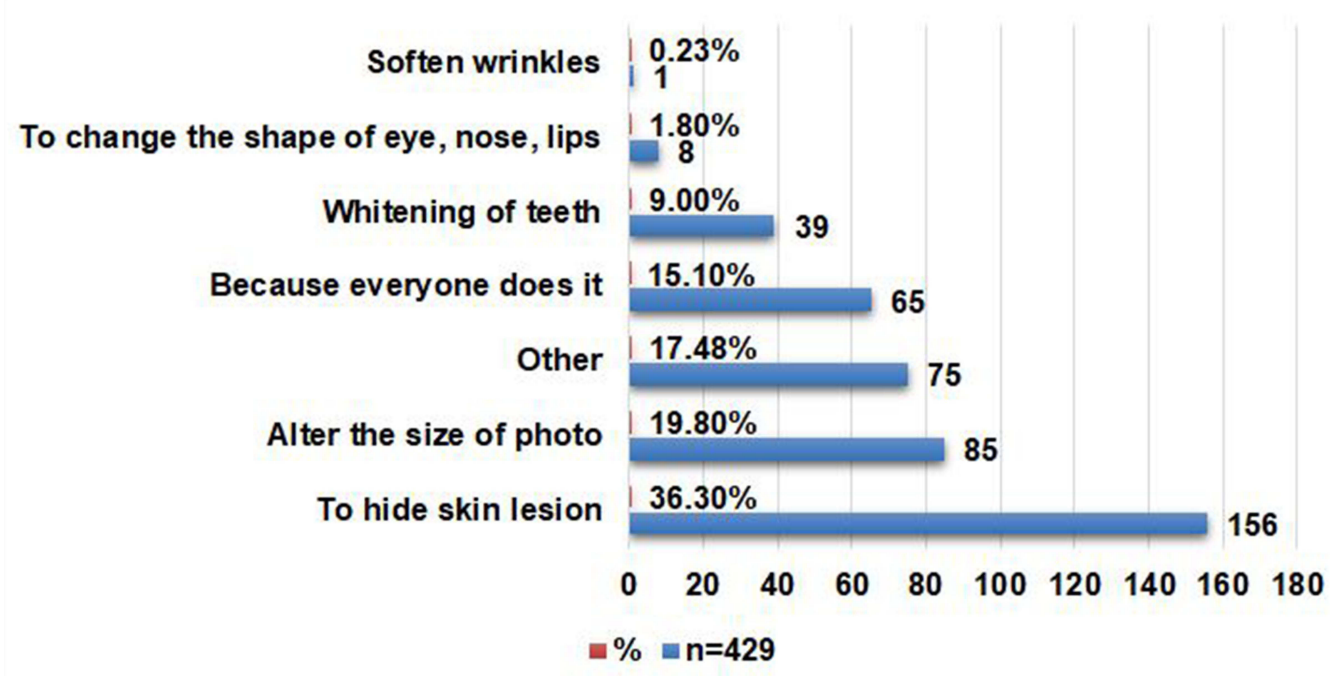

Figure I Reason for editing the photo.

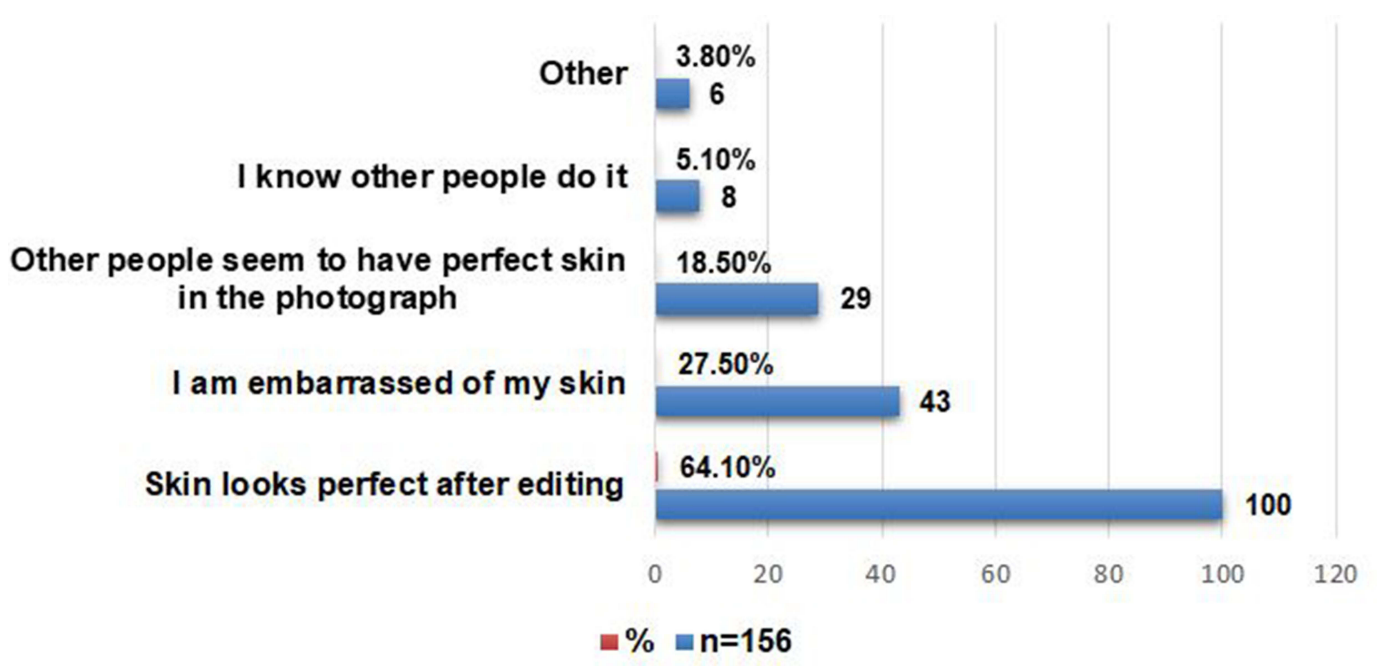

Figure 2 Reason for editing the skin lesion.

motivational factors as a lot and top motivation for using the cosmetic dermatological procedures (Figure 3).

\section{Cosmetic Dermatology Care Seeking Behavior}

Only one-fourth participants had visited a dermatologist before for their cosmetic dermatology care. However, 70\% of the responders were willing to visit a dermatologist in future, if needed. We found that, only $37.4 \%$ of participants preferred to take advice from a dermatologist regarding their cosmetic dermatology care and the majority preferred to seek an opinion from beauty influencers, family, friends and beauticians (Figure 4). When asked about the reason for not taking advice from a dermatologist, the maximum participants felt that it was not needed $(41 \%)$, the services were costly $(32 \%)$ and they could not visit due to their busy schedule (22\%).

\section{Self-Esteem of the Participants}

Almost $90 \%$ of our respondents had high self-esteem.

\section{Association of the Cosmetic} Dermatology Care Seeking Behavior of Social Media Users with Various Variables On multivariate analysis, respondents who were aware of skin care were willing to seek care from a dermatologist than a non-dermatologist. However, Snapchat users; participants who edited $>40 \%$ of photos before posting them on social 
- Least motivation $\quad$ A little bit motivation $=$ Moderate motivation

a lot motivation $\quad$ Top motivation
TO IMPROVE THE QUALITY OF LIFE

FEEL HAPPY OR BETTER OVERALL

LOOK YOUNG OR FRESHER

BE ABLE TO LOOK GOOD WITHOUT MAKEUP

REPAIR DAMAGE OF SKIN

TO LOOK BETTER, PRETTIER OR MORE ATTRACTIVE TO ONESELF

\section{5}

26

26.8

\section{4}

\begin{tabular}{|c|c|c|}
\hline 14 & 33 & 37.6 \\
\hline
\end{tabular}

\begin{tabular}{|l|l|l|l|}
\hline 7.48 .2 & 19.4 & 33.8 & 30.9
\end{tabular}

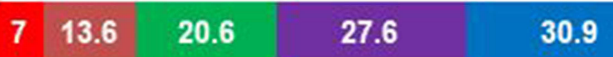

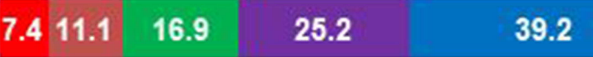

11.

Figure 3 Motivation for using cosmetic dermatology procedure (\%).

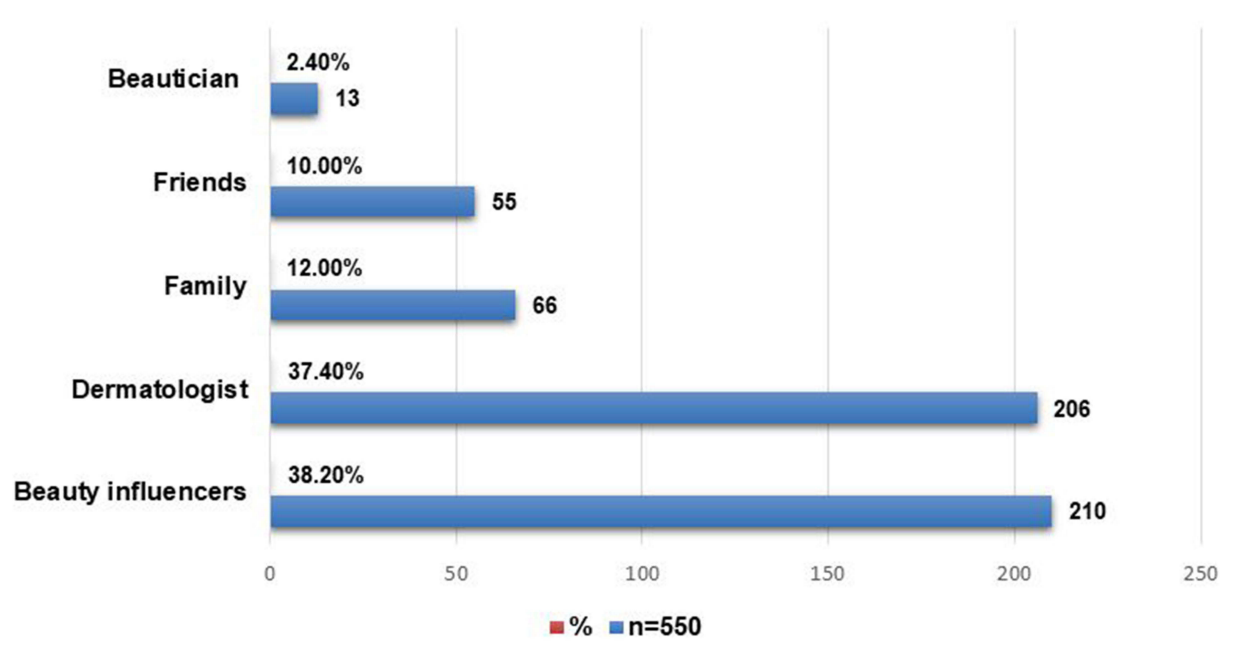

Figure 4 Advice for cosmetic dermatology care.

media; who chose dark circle as the type of skin lesion edited; following others/trends as the reason for editing skin lesion; who were aware of common cosmetic dermatological procedures, had low self-esteem, had never visited a dermatologist and were not willing to visit a dermatologist in future favored to seek care from non-dermatologists. These values were statistically significant (Table 1).

\section{Discussion}

The use of social media and photo-editing applications is in trend more than ever, especially in the younger population. The Holy Trinity of social media; Instagram, Facebook and YouTube has changed the way people perceive and present themselves. And this practice may have significant implications in the field of dermatology.

To our knowledge, this is the first study to evaluate the use of photo-editing applications by young social media users and its effect on cosmetic dermatologic care seeking behavior.

In our study, the maximum numbers of participants using social media (83.6\%) were between 18 and 24 years of age, which is in concordance with the findings of the previous study $^{3}$ as the youth have more inclination towards the use of 


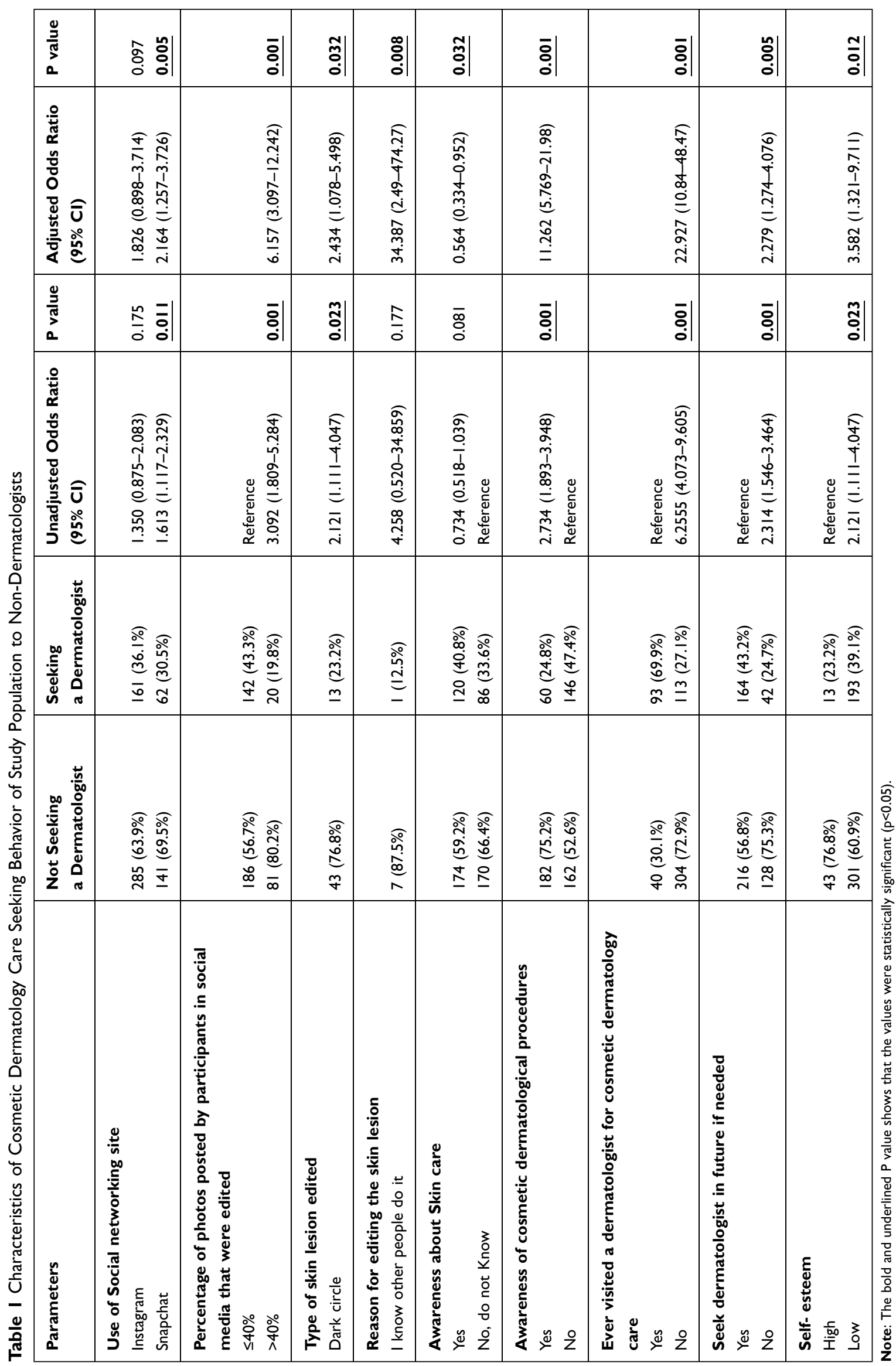


social media. Similar to the study done by Chen et al, ${ }^{5}$ the females outnumbered the males and most of the participants had at least achieved secondary level of education. ${ }^{8}$

According to a survey by Statistica, the most used social networking site in 2020 was Facebook with 2498 million users followed by YouTube, WhatsApp, Facebook Messenger, WeChat and Instagram. ${ }^{14}$ In our study as well, Facebook was the most commonly used site followed by YouTube, Instagram, WhatsApp and Viber. Snapchat was used by $36.9 \%$ of respondents.

Smart insights 2020 found that social media users are now spending an average of 2 hours and 24 minutes per day. ${ }^{15}$ In our study approximately $50 \%$ of participants used social networking sites for more than 3 hours a day. This increase may be due to the current COVID-19 pandemic when people are having more leisure time than before.

Almost all participants had posted a photo of themselves on social media, preferably on Instagram and Facebook, probably a preference for posting photos among close groups. This was contrary to the findings of Williamson et al, where the chosen site for posting photo was twitter. ${ }^{16}$

The photo-editing before posting was found to be very common among social media users in our study. In agreement with our findings Martel et al also observed similar practices in $88.7 \%$ of participants. ${ }^{3}$ Furthermore, concurring to the study by Sheldon et al, the time spent on editing a photo was less than 5 minutes. ${ }^{17}$

Our study observed that hiding skin lesions like, acne or acne scar, pigmentation and dark circles were the most common reason for editing a photograph. These findings are in concordance with the observation of different studies. $^{3-7}$ Moreover; we noted that the majority edited the skin lesion because they felt that their skin looked perfect after editing. Contrary to the present study where embarrassment due to skin lesion was present in less than one-third of the participants, Martel et al reported it as the major reason for photo editing. ${ }^{3}$ Additionally, similar to the study done by Chen et al, ${ }^{5}$ fifty percent of participants were dependent on appearance and had untagged themselves or removed a photo from the social media when the photo was not digitally enhanced or edited to their liking.

In concordance with the findings of similar study, ${ }^{3}$ most of the participants thought that the cosmetic dermatology deals with improving the appearance of skin as well as treatment of skin disease. In the present study, although $44 \%$ participants knew about the cosmetic dermatological procedures, majority knew about acne scarring treatment and hair transplantation. This is in contrast to the findings given by Pathak ${ }^{18}$ where $83 \%$ of participants were aware of cosmetic dermatological procedures with botox being the most popular one. This discordance may be due to the different study population, as health workers were included in the study, who were more aware of the usual cosmetic procedures done in their hospital.

Only one-fourth of our participants had visited a dermatologist for cosmetic dermatology care. Interestingly, Martel et al reported that about $42 \%$ of social media users visited a dermatologist for cosmetic dermatology care. ${ }^{3}$ This may be because, our participants might not have considered their skin problem as severe enough to visit a dermatologist. However, similar to their study, three-fourth of our respondents were willing to visit a dermatologist in future, if required.

A primary motivator for patients seeking cosmetic procedures is the desire to look better in photographs. ${ }^{8}$ The rising trend of pursuing cosmetic procedures based on social media inspiration highlights the need to better understand the patients' motivations to seek cosmetic procedures. In the present study, more than $50 \%$ responded that the motivations to seek cosmetic dermatological care are to look better, or attractive to themselves, repair the damaged skin, look young, look good without make up and improve the quality of life. Three-fourth of the respondents felt that the cosmetic dermatology procedures made them better overall.

Self-esteem describes a person's overall sense of selfworth or personal value. Social media and photo-editing application users had lower self-esteem than non-users in the previous study. ${ }^{6}$ Surprisingly, almost $90 \%$ of our respondents had high self-esteem.

Unfortunately, only $37.4 \%$ of participants preferred to go to the dermatologists for their cosmetic dermatology care while the majority preferred taking advice from nondermatologists. Similar observations were noted by Ross et al, where participants preferred friends, family and social media for advice regarding cosmetic dermatology care. ${ }^{19}$ Another survey showed that $45 \%$ of participants were influenced by social media in seeking dermatology care. ${ }^{20}$

Almost 50\% participants felt that a dermatologist consultation was not needed, $32 \%$ felt that visiting a dermatologist was costly and $22 \%$ participants could not visit a dermatologist due to their busy schedule.

We compared the factors that influenced the young social media users and photo editors to visit the dermatologists or non-dermatologists for seeking cosmetic dermatology care. 
The findings suggest that the respondents who were aware of skin care were willing to seek care from a dermatologist than a non-dermatologist. However, Snapchat users; participants who edited $>40 \%$ of photos before posting them on social media; who chose dark circle as the type of skin lesion edited; following others/trends as the reason for editing skin lesion; who were aware of common cosmetic dermatological procedures, had low self-esteem, had never visited a dermatologist and were not willing to visit a dermatologist in future favored to seek care from non-dermatologists.

Individuals have a wide range of influences in the decision to undergo a cosmetic procedure and social media advertisement is one of them. Though, they may prefer to read patient awareness and education materials written by their doctors on an interactive social media webpage, most of the dermatologists do not have webpages. The beauty vloggers and others are more frequent in posting about the different aesthetic procedures, treatment and results as compared to dermatologists which can be reflected by a simple google search. For an instance, when a google search for the treatment of dark circles is done, most of the search results are about the information provided by the non-dermatologists and only very few research papers or clinical trials responses. Our study highlights the need for more awareness and education resources on social media platforms by dermatologists.

\section{Conclusion}

Our study showed that the majority of social media users and photo-editors were more influenced towards nondermatologist professionals than dermatologists for cosmetic dermatology care. Higher investment in social media and the use of photo-editing might be associated with increased nondermatologist seeking behavior. The dissemination of accurate information on social media by a trained dermatologist or by dermatological society is vital for promoting skin health, dispelling false and potentially harmful information that may be popularized on social media by others.

\section{Acknowledgments}

We would like to thank B.P Koirala Institute of Health Sciences and Department of Dermatology for giving us the opportunity to conduct this research. We also express our gratitude to all the participants for their active participation.

\section{Disclosure}

The authors have no conflicts of interest to declare in this work.

\section{References}

1. Frimmings RE, Polsgrove MJ, Bower GG. Evaluation of a health and fitness social media experience. Am J Health Educ. 2011;42 (4):222-227. doi:10.1080/19325037.2011.10599191

2. Escoffery C, Miner KR, Adame DD, Butler S, McCormick L, Mendell E. Internet use for health information among college students. JAm College Health. 2005;53(4):183-188. doi:10.3200/ JACH.53.4.183-188

3. Martel J, Powell E, Murina A. The effect of Instagram and photo-editing on seeking dermatologic care. J Cosmet Dermatol. 2020;19:1-4.

4. McLean SA, Paxton SJ, Wertheim MJ. Selfies and social media: relationships between self-image editing and photo-investment and body dissatisfaction and dietary restraint. J Eat Disord. 2015;3:O21. doi:10.1186/2050-2974-3-S1-O21

5. Chen J, Ishii M, Bater KL, et al. Association between the use of social media and photograph editing applications, self-esteem, and cosmetic surgery. JAMA Facial Plast Surg. 2019;21(5):361-367. doi:10.1001/jamafacial.2019.0328

6. Rajanala S, Maymone MBC, Vashi NA. Selfies-living in the era of filtered photographs. JAMA Facial Plast Surg. 2018;20(6):443-444. doi:10.1001/jamafacial.2018.0486

7. Fardouly J, Vartanian LR. Negative comparisons about one's appearance mediate the relationship between Facebook usage and body image concerns. Body Image. 2015;12:82-88. doi:10.1016/j. bodyim.2014.10.004

8. Maisel A, Waldman A, Furlan K, et al. Self-reported patient motivations for seeking cosmetic procedures. JAMA Dermatol. 2018;154 (10):1167-1174. doi:10.1001/jamadermatol.2018.235

9. Heughan C, Kanigsberg N, Amdemichael E, Fergusson D, Ammerman D. Canaries in the mineshaft: the dermatology workforce shortage in Eastern Ontario. J Cutan Med Surg. 2008;12(5):217-222. doi:10.2310/7750.2008.07071

10. Patan Academy of Health Sciences. Nepal at a glance. Available from: http://www.pahs.edu.np/about/about-nepal/. Accessed September 13, 2021.

11. Agrawal S. Cosmetic/surgical dermatology: should it be an integrated part of medical dermatology? KUMJ. 2016;14(3):190-191.

12. Sharecast Initiative Nepal. Nepal media survery 2019. Available from: http://www.sharecast.org.np/nepal-media-survey-2019-findings /31. Accessed September 13, 2021.

13. Rosenberg M. Society and the Adolescent Self-Image. Revised ed. Middletown, CT: Wesleyan University Press; 1989.

14. Statistica Research Department. Number of global social network users 2017-2025| statista". Statista. Available from: https://www.sta tistica.com/statistics/278414/number-of-worldwide-social-networkusers/. Accessed September 13, 2021.

15. Smart Insights. Global social media statistics research summary 2021. Available from: https://www.smartinsights.com/social-mediamarketing/social-media-strategy/new-global-social-media-research/. Accessed September 13, 2021.

16. Williamson P, Stohlman T, Polinsky H. Me, my selfie and I: a survey of self-disclosure motivations on social media. IAFOR J Cult Stud. 2017;2(2):78-89.

17. Sheldon P, Bryant K, Sheldon P, Bryant K. Instagram: motives for its use and relationship to narcissism and contextual age. Comput Hum Behav. 2016;58:89-97. doi:10.1016/j.chb.2015.12.059 
18. Pathak D. Awareness of cosmetic dermatology procedures among health workers in a tertiary care hospital. Indian Dermatol Online J. 2019;10(2):139-143.

19. Ross NA, Todd Q, Saedi N. Patient seeking behaviors and online person media's role in cosmetic dermatology. Dermatol Surg. 2015;41(2):269-276. doi:10.1097/DSS.0000000000000267
20. PWC Health Research Institute. Social media "likes" healthcare: from marketing to social business; 2012. Available from: https:// www.pwc.com/us/en/health-industries/healthresearchinstitute/publica tions/pdf/health-care-social-media-report.pdf. Accessed June 4, 2019.

\section{Publish your work in this journal}

Clinical, Cosmetic and Investigational Dermatology is an international, peer-reviewed, open access, online journal that focuses on the latest clinical and experimental research in all aspects of skin disease and cosmetic interventions. This journal is indexed on CAS
The manuscript management system is completely online and includes a very quick and fair peer-review system, which is all easy to use. Visit http://www.dovepress.com/testimonials.php to read real quotes from published authors. 